Mod. Phys. Lett. A, submitted for publication

(C) World Scientific Publishing Company

\title{
KdV ADIABATIC INDEX SOLITONS IN BAROTROPIC OPEN FRW COSMOLOGIES
}

\author{
HARET C. ROSU \\ Instituto de Física, Universidad de Guanajuato, Apdo Postal E-143, 37150 León, Gto, Mexico
}

\begin{abstract}
Applying standard mathematical methods, it is explicitly shown how the Riccati equation for the Hubble parameter $H(\eta)$ of barotropic open FRW cosmologies is connected with a Korteweg-de Vries equation for adiabatic index solitons. It is also shown how one can embed a discrete sequence of adiabatic indices of the type $n^{2}\left(\frac{3}{2} \gamma-1\right)^{2}(\gamma \neq 2 / 3)$ in the sech FRW adiabatic index soliton.
\end{abstract}

\section{1 - Riccati equation for barotropic FRW cosmologies}

Recently, Faraoni, 1 showed that the equations describing barotropic FRW cosmologies can be combined in a simple Riccati equation leading in a much easier way to the textbook solutions for the FRW scale factors. Faraoni obtained the following cosmological Riccati equation

$$
\frac{d H}{d \eta}=-c H^{2}-\kappa c
$$

for the log derivative of the FRW scale factor, the famous Hubble parameter $H(\eta)=$ $\frac{d a / d \eta}{a}$. The independent variable is the conformal time $\eta, c$ is simply related to the adiabatic index of the cosmological fluid, $c=\frac{3}{2} \gamma-1$ which was assumed constant by Faraoni, and $\kappa=0, \pm 1$ is the curvature index of the flat, closed, open FRW universe, respectively. For the mathematical considerations of this work we are more interested in the open cosmologies $\kappa=-1$. The flat case is special and has been discussed in a previous work 1 whereas the case $\kappa=+1$ will be commented in subsection 3.2 herein.

We notice that from the mathematical point of view, Faraoni's FRW Riccati equation being of constant coefficients is directly integrable. The solutions are

$$
H^{+}=-\tan \left(c \eta-\phi_{+}\right), \quad H^{-}=\tanh \left(c \eta-\phi_{-}\right),
$$

for the closed and open FRW universes, respectively. Of course, depending on the initial conditions, the solutions can be written in terms of the corresponding cofunctions as well. The minus sign in front of the phases has been chosen for later convenience. 


\section{2 - Cosmological Korteweg de Vries equation}

We now pay special attention to the $\kappa=-1$ case for which a connection with the $\mathrm{KdV}$ equation is possible. Employing the well-known change of function $\mathrm{H}^{-}=$ $\frac{1}{c} \frac{w^{\prime}}{w}$ one can pass from the nonlinear Riccati equation to the linear second-order differential equation

$$
w^{\prime \prime}-c^{2} w=0,
$$

where the prime means derivation with respect to $\eta$. This equation can be factorized

$$
A_{1} A_{2} w=0
$$

by means of the operators

$$
A_{1}=\left(\frac{d}{d \eta}+W\right), \quad A_{2}=\left(\frac{d}{d \eta}-W\right)
$$

where $W=c H^{-}$. The general linear solution (the general zero mode) is of the form

$$
w(\eta, T)=\alpha(T) e^{c \eta}+\beta(T) e^{-c \eta},
$$

where we have assumed that the superposition constants are functions of a new coordinate $T$ that is independent of $\eta$. It is easy to show the relationship between the two superposition constants and the phase $\phi_{-}$

$$
\phi_{-}(T)=\frac{1}{2} \ln \beta / \alpha .
$$

Inverting as in SUSY QM the order of application of the factorization operators, one gets an equation of the type

$$
A_{2} A_{1} u=0 \quad \text { or } \quad u^{\prime \prime}+c_{1}^{2}(\eta, T) u=0,
$$

where $c_{1}(\eta, T)$ is the sech soliton with the asymptotic wings going to $c^{2}$

$$
c_{1}^{2}(\eta, T)=c^{2}-2 c^{2} \operatorname{sech}^{2}\left(c \eta-\phi_{-}(T)\right) .
$$

It is now easy to apply basic results from the $\mathrm{KdV}$ mathematics allowing to interpret $c_{1}^{2}$ as a single sech soliton of the following $\mathrm{KdV}$ equation 3

$$
\frac{d C}{d T}-6 C \frac{d C}{d \eta}+\frac{d^{3} C}{d \eta^{3}}=0
$$

if the condition $\frac{d \phi_{-}}{d T}=4 c^{3}$ is imposed. Moreover, by choosing initial conditions at $T=0$, one can see that $T$ is the evolution variable whereas the conformal time plays a role similar to a spatial variable 


\section{3 - SUSY recursive procedure}

3.1 - The scheme presented in the previous section can be iterated in a simple and well-known manner. profile and take $\phi_{-}=0$ for simplicity. Thus, we start with a 'quantum' mechanical system in a constant 'potential' $c^{2}$ and relate it to a Schrödinger-type equation in the conformal time domain which has a fundamental 'frequency' at $-c^{2}$. We solve the "fermionic" Riccati equation i.e.,

$$
W_{1}^{2}-W_{1}^{\prime}-c^{2}=0
$$

to find Witten's superpotential $W_{1}(\eta)=-c \tanh [c \eta]$ and next go to the "bosonic" Riccati equation

$$
W_{1}^{2}+W_{1}^{\prime}+c_{1}^{2}(\eta)-c^{2}=0,
$$

in order to get $c_{1}^{2}(\eta)=-2 c^{2} \operatorname{sech}^{2}[c \eta]$. Moreover, one can write the Schrödinger equation corresponding to the "bosonic" Riccati equation as follows

$$
-\tilde{y}^{\prime \prime}+c_{1}^{2}(\eta) \tilde{y}=-c^{2} \tilde{y}
$$

with the solution $\tilde{y} \propto c \operatorname{sech}(c \eta)$. The physical picture is that of a sech soliton profile containing a single mode self-trapped at $-c^{2}$ within the frequency pulse. One can employ the scheme recursively to get several localized modes embedded in the soliton profile. Indeed, suppose we would like to introduce $N$ adiabatic indices of the type $c_{n}^{2}=-n^{2} c^{2}, n=1, \ldots N$ in the sech pulse. Then, one has to solve the sequence of equations

$$
\begin{gathered}
W_{n}^{2}-W_{n}^{\prime}=c_{n-1}^{2}+n^{2} c^{2}, \\
W_{n}^{2}+W_{n}^{\prime}=c_{n}^{2}+n^{2} c^{2},
\end{gathered}
$$

inductively for $n=1 \ldots N$. $1 \ldots N$ is of the form $c_{N}^{2}(\eta)=-N(N+1) c^{2} \operatorname{sech}^{2}(c \eta)$. The corresponding modes can be written in a compact form as follows

$$
\tilde{y}_{n}(\eta ; N) \approx A^{\dagger}(\eta ; N) A^{\dagger}(\eta ; N-1) A^{\dagger}(\eta ; N-2) \ldots A^{\dagger}(\eta ; N-n+2) \operatorname{sech}^{N-n+1} c \eta,
$$

i.e., by applying the first-order operators $A^{\dagger}\left(\eta ; a_{n}\right)=-\frac{d}{d \eta}-a_{n} c \tanh (c \eta)$, where $a_{n}=N-n$, onto the "ground state" sech mode.

3.2 - For the case $\kappa=1$ the "fermionic" Riccati equation

$$
W_{1}^{2}-W_{1}^{\prime}+c^{2}=0
$$

leads to the solution $W_{1}=c \tan (c \eta)$ and from the "bosonic" Riccati equation

$$
W_{1}^{2}+W_{1}^{\prime}+c_{1}^{2}+c^{2}=0,
$$


one will find $c_{1}^{2}(\eta)=2 c^{2} \sec ^{2}(c \eta)$. Consequently, the Schrödinger-like equation

$$
-\tilde{y}^{\prime \prime}+c_{1}^{2}(\eta) \tilde{y}=c^{2} \tilde{y}
$$

has solutions of the type $\tilde{y} \propto c \sec (c \eta)$, which is not of the localized soliton type, and therefore the approach leads to unphysical results.

\section{4 - Conclusion}

A cosmological KdV equation for adiabatic index solitons in the realm of barotropic FRW models has been introduced in this work. A simple recursive nonrelativistic SUSY scheme has been also applied to show that in the barotropic soliton profile one can embed a discrete sequence of adiabatic indices. Each eigenmode of the soliton corresponds to one of the embedded adiabatic indices.

KdVc.tex 


\section{References}

1. V. Faraoni, Am. J. Phys. 67, 732 (1999) physics/9901006. See also, J.A.S. Lima, Am. J. Phys., to appear, astro-ph/0109215. For supersymmetric approach with application to cosmological acceleration, see H.C. Rosu, Mod. Phys. Lett. A 15, 979 (2000) grqc/0003108.

2. H.C. Rosu, Mod. Phys. Lett. A 16, 1147 (2001) gr-qc/0003108.

3. It seems that a water $\mathrm{KdV}$ sech soliton has been first observed in a shallow drainage channel during the month of August 1834 by J.S. Russell, who followed it on horseback in a chase of one or two miles. The KdV equation has been first obtained in 1895 .

4. Using the direction of propagation as an evolution coordinate is a well-known procedure for pulse propagation in nonlinear materials and in waveguide physics, see, e.g., G. Fibich, V.M. Malkin, G.C. Papanicolau, Phys. Rev. A 52, 4218 (1995).

5. J.L. Rosner, Ann. Phys. 200, 101 (1990); W. Kwong and J.L. Rosner, Prog. Theor. Phys. Suppl. 86, 366 (1986).

6. H. Rosu, J.L. Romero, J. Socorro, Nuovo Cimento B 113, 549 (1998) physics/9707012. 